\title{
Do SARS-CoV-2 Infection (COVID-19) and the Medications Administered for Its Treatment Impair Testicular Functions?
}

\author{
Abdullah Gul Salim Zengin Gokce Dundar Murat Ozturk \\ University of Health Sciences Turkey, Bursa Yuksek Ihtisas Training and Research Hospital, Bursa, Turkey
}

\section{Keywords}

Androgens · COVID-19 · SARS Coronavirus $2 \cdot$ Semen

analysis $\cdot$ Spermatogenesis

\begin{abstract}
Purpose: Severe acute respiratory syndrome coronavirus 2 (SARS-CoV-2) is transmitted primarily via respiratory droplets and enters host cells through angiotensin-converting enzyme 2 (ACE-2) receptors. ACE-2 receptors have been identified in many tissues including testes. The aim of the study has been to investigate the long-term effects of SARSCoV-2 infection (COVID-19) and its relative treatment on male reproductive health. Methods: Cross-sectional analysis has been performed on 49 recovered COVID-19 patients who had semen analysis prior to the COVID-19 pandemic. Those who had a recovery time lag of at least 3 months have been re-examined, and 29 eligible patients with no andrological problems have been enrolled in the study. Following a detailed physical examination and retrieval of medical history, the values of semen analysis and serum sex hormone parameters have been collected and compared before and after COVID-19 infection. The $p$ value of $<0.05$ has been considered significant. Results: The average age of the 29 pa-
\end{abstract}

tients has been $31.21 \pm 5.48$ (range: 18-41) years. Favipiravir has been co-administered with hydroxychloroquine in 17 patients, while the remaining 12 received favipiravir treatment without hydroxychloroquine. The average time between clinical recovery from COVID-19 and collection of semen has been $4.52 \pm 1.36$ (range: 3-8) months. Before and after COVID-19, serum follicle-stimulating hormone, luteinizing hormone, total testosterone, and prolactin levels, as well as all semen parameters, have been comparable. Conclusion: Our study demonstrated that COVID-19 and its treatment with favipiravir and hydroxychloroquine did not affect spermatogenesis and serum androgen levels in the long-term period. Further clinical studies with larger sample size are needed to confirm and support our findings.

๑) 2021 S. Karger AG, Basel

\section{Introduction}

COVID-19, a severe acute respiratory syndrome coronavirus 2 (SARS-CoV-2) infection, was first observed in China in December 2019 (Wuhan City, Hubei Province) [1]. COVID-19 was declared as a pandemic by the World Health Organization (WHO) in March 2020 due to its rap- 
id spread across the globe. According to the WHO's situation report released on April 20, 2021, it has infected $>140$ million patients worldwide and killed $>3$ million patients.

SARS-CoV-2 is transmitted primarily via respiratory droplets and enters host cells through angiotensin-converting enzyme 2 (ACE-2) receptors [2]. ACE-2 receptors have been identified in a variety of processes, including the cardiovascular, gastrointestinal, neuroendocrine, genitourinary, and respiratory systems $[2,3]$. Human testis is one of the organs that contain these receptors $[3,4]$. As a result, it is assumed that SARS-CoV-2 may have a negative impact on the male reproductive tract, and numerous studies have been conducted to determine whether the virus is present in semen and how it affects spermatogenesis [5-10]. However, all of these studies have been conducted during the disease's acute or subacute phase. It is well-known that it can take up to 76 days for new semen to mature [11]. According to our review of the literature, there is a knowledge gap about the long-term effects of SARS-CoV-2 infection and treatment on the male reproductive system, including spermatogenesis and serum sex hormone parameters. By comparing the results of semen analysis and serum sex hormone levels of men before SARS-CoV-2 infection with those after at least 3 months of recovery from SARS-CoV-2 infection, we aimed to close this significant gap.

\section{Materials and Methods}

In accordance with the Helsinki Declaration, our study has been conducted after receiving approval from the Turkish Ministry of Health and the Ethical Committee of Bursa Yuksek Ihtisas Training and Research Hospital (IRB No. 2011-KAEK-25 2021/01-18). In addition, we obtained written informed consent from all patients who agreed to participate in this study. Our hospital database has been used to search for patients who have been admitted to our outpatient clinic for infertility investigations between September 2019 and March 11, 2020, when the first case of COVID-19 has been reported in Turkey. We identified 476 men who have been evaluated using semen analysis. Using our country's public health management system database, we then identified 49 patients among them who had COVID-19 between March 11, 2020 and March 31, 2021. The SARS-CoV-2 nucleic acid test in the pharyngeal swap has been used to diagnose COVID-19. We have reached out to the COVID-19-positive patients and invited those who had recovered from COVID-19 at least 3 months ago to come to our hospital for re-examination. A comprehensive clinical history and physical examination have been performed, as well as evaluation of patients' semen analysis and urinalysis, hormonal determinations, genetic testing, and scrotal ultrasound as needed. We also documented the laboratory findings, such as complete blood count, C-reactive protein levels, and D-dimer values, as well as radiological examinations for the lungs, such as chest X-rays and/or computed tomography imaging, that have been performed on patients admitted to the
Table 1. Features of the study population $(n=29)$

\begin{tabular}{lcc}
\hline Characteristic & Mean \pm SD & Range (min-max) \\
\hline Age, years & $31.21 \pm 5.48$ & $18-41$ \\
$\mathrm{BMI}, \mathrm{kg} / \mathrm{m}^{2}$ & $27.05 \pm 2.34$ & $22-31$ \\
$\mathrm{Hgb}, \mathrm{g} / \mathrm{dL}$ & $15.41 \pm 1.14$ & $12.6-17$ \\
$\mathrm{WBC}, \times 10^{3} / \mu \mathrm{L}$ & $6.90 \pm 2.16$ & $4.31-12.86$ \\
$\mathrm{Lymphocyte} / \mu \mathrm{L}$ & $1.91 \pm 0.88$ & $0.64-4.04$ \\
Platelet, $\times 10^{3} / \mu \mathrm{L}$ & $258.57 \pm 55.75$ & $165-366$ \\
$\mathrm{CRP}, \mathrm{mg} / \mathrm{L}$ & $6.75 \pm 7.80$ & $3.10-33.11$ \\
D-dimer, $\mu \mathrm{g} / \mathrm{L}$ & $0.26 \pm 0.07$ & $0.19-0.40$ \\
\hline
\end{tabular}

SD, standard deviation; BMI, body mass index; WBC, white blood cell; CRP, C-reactive protein.

emergency department with symptoms suggestive of COVID-19. The analysis of ejaculated semen has been carried out in accordance with WHO recommendations and principles [12]. Between 9:00 and 10:00 a.m., fasting serum samples have been collected in order to measure total testosterone (TT), follicle-stimulating hormone (FSH), prolactin, and luteinizing hormone ( $\mathrm{LH})$.

The following patients met the inclusion criteria for the current study: they were $\geq 18$ years old, had laboratory-confirmed COVID-19, and had survived at least 3 months after treatment and recovery. Patients with endocrinologic and genetic disorders, chronic diseases (cardiovascular disorders, hypertension, diabetes, etc.), or congenital or acquired urogenital anomalies, with the presence of known causes of infertility such as undescended testis, a varicocele, or a previous history of surgery were excluded from this study. Furthermore, patients with acute urogenital tract infections or a history of epididymo-orchitis, a history of any cancer, or who had undergone any type of treatment that could influence spermatogenesis and the hypothalamo-pituitary-gonadal axis were excluded from the study.

For data analysis, we used the IBM SPSS statistics program, version 21.0 (IBM Corp., Chicago, IL, USA). The variables have been presented as means and standard deviations, as well as numbers $(n)$ and percentages (\%). We calculated the number of participants required for our study design using the $\mathrm{G}^{*}$ Power 3.1 program, and according to power analysis with a 0.80 power value, a 0.05 error and a 0.56 effect size based on the study of Temiz et al. [9], 28 patients have been sufficient for statistical analysis. The KolmogorovSmirnov test has been used to determine whether the data distribution had been normal. For the comparison of variables before and after COVID-19 infection, the Wilcoxon signed-rank test has been used. A statistically significant $p$ value of 0.05 has been used.

\section{Results}

After applying the exclusion criteria to the volunteers, 29 eligible male patients with a mean age of $31.21 \pm 5.48$ years have been enrolled in the current study. Table $1 \mathrm{dem}-$ onstrates the characteristics of the study population as well as the results of blood samples collected during an exami- 
Table 2. Comparison of the values before and after COVID-19 infection and its treatment

\begin{tabular}{lccc}
\hline Variable & $\begin{array}{l}\text { Before COVID-19 } \\
(\text { mean } \pm \text { SD) }\end{array}$ & $\begin{array}{c}\text { After COVID-19 } \\
\text { and its treatment } \\
(\text { mean } \pm \text { SD) }\end{array}$ & $p$ value \\
& & & \\
\hline Serum sex hormone parameters & $3.92 \pm 1.97$ & $4.15 \pm 2.22$ & 0.161 \\
$\quad$ FSH, IU/L & $4.46 \pm 1.62$ & $4.62 \pm 1.68$ & 0.263 \\
LH, IU/L & $423 \pm 100$ & $432 \pm 123$ & 0.779 \\
TT, ng/dL & $9.43 \pm 2.71$ & $9.32 \pm 2.85$ & 0.944 \\
PRL, ng/mL & & & \\
Semen parameters & $2.23 \pm 1.11$ & $2.58 \pm 1.01$ & 0.138 \\
Semen volume, $\mathrm{mL}$ & $39.67 \pm 40.45$ & $47.52 \pm 60.84$ & 0.573 \\
$\quad$ Sperm count, $\times 10^{6} / \mathrm{mL}$ & $77.88 \pm 75.61$ & $100.80 \pm 107.01$ & 0.314 \\
Total sperm count, $\times 10^{6}$ & $26.62 \pm 12.59$ & $29.24 \pm 15.49$ & 0.107 \\
$\quad$ Progressive motility, $\%$ & $31.23 \pm 15.06$ & $33.97 \pm 19.07$ & 0.334 \\
$\quad$ Total motility, $\%$ & & & \\
\hline
\end{tabular}

FSH, follicle-stimulating hormone; LH, luteinizing hormone; TT, total testosterone; PRL, prolactin. nation at the emergency department. The mean time between clinical recovery from COVID-19 infection and semen collection was $4.52 \pm 1.36$ (range: $3-8$ ) months. Furthermore, the mean time between semen samples before and after COVID-19 infection was $8.05 \pm 1.26$ (range: $6-11)$ months. Based on radiological images, 3 of the 29 patients (10.3\%) had COVID-19-associated pulmonary infiltration, and 2 of them (6.9\%) have been hospitalized. The average hospital stay has been $6.5 \pm 0.7$ days, with one hospital staying for 6 days and the other staying for 7 days. Favipiravir 1,600 mg b.i.d. for the first day, $600 \mathrm{mg}$ b.i.d. for the next 9 days, and hydroxychloroquine $200 \mathrm{mg}$ b.i.d. for 10 days have been administered daily to hospitalized patients. Favipiravir has been co-administered with hydroxychloroquine to 15 home care patients, while the remaining 12 received favipiravir treatment without hydroxychloroquine for 5 days. In addition, all patients received subcutaneous enoxaparin at a dose of 4,000 IU once daily for thromboprophylaxis. Comparisons of sperm values and serum sex hormone levels before and after COVID-19 infection have been all similar (demonstrated in Table 2). Conversely, when the patients have been divided into subgroups as hospitalized or nonhospitalized individuals, as well as based on the various treatment protocols listed above, they have not been statistically different.

\section{Discussion}

Coronaviruses have been identified in 1965 and have many different species, one of which is SARS-CoV [12, 13]. It has been reported that SARS-CoV-1 is associated with testicular damage, orchitis, germ cell destruction, and spermatogenesis dysfunction [14]. Because SARSCoV-2 is genomically similar to SARS-CoV-1 and has a relationship with ACE-2 receptors, which are found in many tissues including the testes, many studies have been conducted on the presence of SARS-CoV-2 in semen samples, sexual transmission via seminal fluid, and potential effects on male reproductive health $[5-10,15,16]$. Recently published studies on SARS-CoV-2 and semen have revealed some contradictory results $[5,6,9,17-19]$. While $\mathrm{Li}$ et al. [5] discovered the presence of SARS-CoV-2 RNA in the ejaculated semen of $6(15.8 \%)$ participants in their study, others did not find in their cohorts [6, 9, 17-19].

Spermatogenesis in SARS-CoV-2 patients, on the other hand, has been researched in a few studies $[6,8,9]$. Holtmann et al. [6] researched 34 men, including 20 COVID-19 patients (18 recovered and 2 active) and 14 healthy participants. They classified the recovered COVID-19 patients as mild $(n=14)$ who received home care and moderate $(n=$ 4) who were hospitalized while infected. In their study, the average length of hospitalization has been $9.2 \pm 5.3$ days [6] Only 2 patients with COVID-19 have been hospitalized in our study, and the average hospital stay was $6.5 \pm 0.7$ days. Our cohort's hospitalization rate and days have been lower than theirs, with an average of $6.9 \%$ versus $22.2 \%$ and 6.5 versus 9.2, respectively. When Holtmann et al. [6] compared the results of mild and moderate infection, they discovered that the moderate group had a significantly lower semen count and motility. Furthermore, all semen parameters have been comparable in the control group and COVID-19 patients with mild symptoms [6]. Our study, which used a different methodology, discovered that semen val- 
ues before and after COVID-19 infection in the same patients did not differ. While they have been evaluated as independent groups with potential confounders, we examined the same patients as dependent groups with no confounders. In addition, the mean time between the end of COVID-19 symptoms and the collection of a semen sample in our study has been $4.52 \pm 1.36$ months, ranging from 3 to 8 months, whereas the time frame between clinical recovery from COVID-19 infection and semen collection in their study was 32.7 days, ranging from 8 to 54 days in total and $34.9 \pm 11.7$ and $25.5 \pm 8.3$ days in mild and moderate patients, respectively. Considering the duration of spermatogenetic process, their study did not allow for the formation of new spermatozoa. Li et al. [8] conducted a study in China investigating the possible changes in autopsied testicular specimens of patients died due to COVID-19 $(n=6)$ and prostate cancer $(n=6)$. In comparison to control patients with prostate cancer, they discovered impaired spermatogenesis and a higher accumulation of $\mathrm{T}$ lymphocytes and macrophages in the specimens [8]. However, factors such as hypertension, steroid treatment, fever, and acute infection have not been taken into consideration in their study which might cause these alterations in deceased COVID-19 patients [8]. They also examined at 23 male COVID-19 patients and 22 age-matched controls and discovered that COVID-19 patients had semen concentrations and higher levels of proinflammatory cytokines than controls [8]. This disparity could be attributed to possible selection bias, the presence of fever, and acute infection. It is well-known that fever, which is common in viral diseases, can harm spermatogenesis [20]. Similar to the study of Holtmann et al. [6], the duration required for the maturation of a new spermatogonial cell was not provided in Honggang's study [8]. Temiz et al. [9] investigated the impact of COVID-19 along with hydroxychloroquine and azithromycin on semen quality and androgen hormones in a prospective study with 3 groups such as highly suspected COVID-19 patients $(n=10)$, treated COVID-19 patients for 5 days $(n=10)$, and controls $(n=10)$. Many sperm parameters were similar across all groups, but sperm morphology has been reduced in COVID-19 patients. They concluded that the cause of the morphological disturbance was fever and that COVID-19 medications had no effect on spermatogenesis in the short term [9]. Similarly, favipiravir and hydroxychloroquine administrations in the current study had no long-term effect on gonadal functions. The antiprotease activity of hydroxychloroquine has been shown in the literature to benefit the development of the blood-testis barrier [21], while there is no evidence about the impact of favipiravir on testicular functions [22]. To the best of our knowledge, this is the first study to investigate the long-term effects of these medications and of SARS$\mathrm{CoV}-2$ infection on the male reproductive system.

The hypothalamo-pituitary-gonadal axis is another critical issue in male reproductive health. Ma et al. [23] reported that COVID-19 patients had increased LH and prolactin levels as well as similarly FSH, TT, and estradiol levels compared to the healthy group in a study involving 81 COVID-19 male patients and 100 age-matched healthy men as the control group. They concluded that elevated LH and normal TT levels in COVID-19 patients could be associated with early stage hypogonadism or a wide range of TT in humans [23]. Another study, conducted in Italy by Rastrelli et al. [24], discovered that COVID-19 patients had low TT levels, which have been negatively correlated with disease severity. A nonpeer-reviewed retrospective study demonstrated that many male patients with COVID-19 had low TT levels during acute infection [25]. Temiz et al. [9] discovered in their study that patients with symptoms suggestive of COVID-19 had lower TT, FSH, and LH levels than COVID-19 negative controls. All sex hormone levels however have been within the normal range and comparable between the control and treated groups [9]. According to our findings, both SARS-CoV-2 infection and treatment with favipiravir and hydroxychloroquine did not appear to change male sex hormone levels over time.

Despite the current study's significant and novel contributions to the literature, it has several limitations. Besides, despite a sufficient number of patients based on the power analysis, it was limited by a relatively small sample size. Second, we did not assess whether SARS-CoV-2 was present in the semen over time.

\section{Conclusion}

Our findings indicate that SARS-CoV-2 infection and treatment with favipiravir and hydroxychloroquine had no long-term effect on male reproductive health. This study however needs to be validated by well-designed large-scale clinical trials.

\section{Statement of Ethics}

In accordance with the 1964 Helsinki Declaration, our study has been conducted after receiving approval from the Turkish Ministry of Health and the Ethical Committee of Bursa Yuksek Ihtisas Training and Research Hospital (IRB no: 2011-KAEK-25 2021/01-18). Informed written consent was obtained from all individual participants included in the study. 


\section{Conflict of Interest Statement}

All authors declare that they have no conflict of interest.

\section{Funding Sources}

This research did not receive any specific support from funding agencies in the public, commercial, or not-for-profit sectors.

\section{Author Contributions}

Research conception and design: Abdullah Gul, Salim Zengin, Gokce Dundar, and Murat Ozturk. Data acquisition: Salim Zengin. Statistical analysis: Abdullah Gul and Salim Zengin. Data analysis and interpretation: Abdullah Gul and Gokce Dundar. Drafting of the manuscript: Abdullah Gul. Critical revision of the manuscript: Abdullah Gul and Gokce Dundar. Obtaining funding: none. Administrative, technical, or material support: all the authors. Supervision: Abdullah Gul and Murat Ozturk. Approval of the final manuscript: all the authors.

\section{References}

1 Zhu N, Zhang D, Wang W, Li X, Yang B, Song $\mathrm{J}$, et al. A novel coronavirus from patients with pneumonia in China, 2019. N Eng J Med. 2020;382(8):727-33.

2 Zhang H, Penninger JM, Li Y, Zhong N, Slutsky AS. Angiotensin-converting enzyme 2 (ACE2) as a SARS-CoV-2 receptor: molecular mechanisms and potential therapeutic target. Intensive Care Med. 2020;46(4):586-90.

3 Li MY, Li L, Zhang Y, Wang XS. Expression of the SARS-CoV-2 cell receptor gene ACE2 in a wide variety of human tissues. Infect Dis Poverty. 2020;9(1):45.

4 Fan C, Li K, Ding Y, Lu WL, Wang J. ACE2 expression in kidney and testis may cause kidney and testis damage after 2019-nCoV infection. MedRxiv. 2020.

5 Li D, Jin M, Bao P, Zhao W, Zhang S. Clinical characteristics and results of semen tests among men with coronavirus disease 2019 . JAMA Netw Open. 2020;3(5):e208292.

6 Holtmann N, Edimiris P, Andree M, Doehmen C, Baston-Buest D, Adams O, et al. Assessment of SARS-CoV-2 in human semen-a cohort study. Fertil Steril. 2020;114(2):233-8.

7 Khalili MA, Leisegang K, Majzoub A, Finelli R, Panner Selvam MK, Henkel R, et al. Male fertility and the COVID-19 pandemic: systematic review of the literature. World J Mens Health. 2020;38(4):506-20.

$8 \mathrm{Li} \mathrm{H}$, Xiao X, Zhang J, Zafar MI, Wu C, Long $\mathrm{Y}$, et al. Impaired spermatogenesis in COVID-19 patients. EClinicalMedicine. 2020;28: 100604.

9 Temiz MZ, Dincer MM, Hacibey I, Yazar RO, Celik C, Kucuk SH, et al. Investigation of SARS-CoV-2 in semen samples and the ef- fects of COVID-19 on male sexual health by using semen analysis and serum male hormone profile: a cross-sectional, pilot study. Andrologia. 2021;53(2):e13912.

10 Ma L, Xie W, Li D, Shi L, Ye G, Mao Y, et al. Evaluation of sex-related hormones and semen characteristics in reproductive-aged male COVID-19 patients. J Med Virol. 2021 Jan;93(1):456.

11 Neto FTL, Bach PV, Najari BB, Li PS, Goldstein M. Spermatogenesis in humans and its affecting factors. Semin Cell Dev Biol. 2016; 59:10-26.

12 Tyrrell DA, Bynoe ML. Cultivation of a novel type of common-cold virus in organ cultures. Br Med J. 1965;1(5448):1467-70.

13 de Wit E, van Doremalen N, Falzarano D, Munster VJ. SARS and MERS: recent insights into emerging coronaviruses. Nat Rev Microbiol. 2016;14(8):523-34.

14 Xu J, Qi L, Chi X, Yang J, Wei X, Gong E, et al. Orchitis: a complication of severe acute respiratory syndrome (SARS). Biol Reprod. 2006;74(2):410-6.

15 Groner MF, de Carvalho RC, Camillo J, Ferreira PRA, Fraietta R. Effects of Covid-19 on male reproductive system. Int Braz J Urol. 2021;47(1):185-90.

16 Patel KP, Vunnam SR, Patel PA, Krill KL, Korbitz PM, Gallagher JP, et al. Transmission of SARS-CoV-2: an update of current literature. Eur J Clin Microbiol Infect Dis. 2020; 39(11):2005-11.

17 Pavone C, Giammanco GM, Baiamonte D, Pinelli M, Bonura C, Montalbano M, et al. Italian males recovering from mild $\mathrm{CO}-$ VID-19 show no evidence of SARS-CoV-2 in semen despite prolonged nasopharyngeal swab positivity. Int J Impot Res. 2020;32(5): $560-2$.

18 Song C, Wang Y, Li W, Hu B, Chen G, Xia P, et al. Absence of 2019 novel coronavirus in semen and testes of COVID-19 patients. Biol Reprod. 2020;103(1):4-6.

19 Pan F, Xiao X, Guo J, Song Y, Li H, Patel DP, et al. No evidence of severe acute respiratory syndrome-coronavirus 2 in semen of males recovering from coronavirus disease 2019 Fertil Steril. 2020;113(6):1135-9.

20 Carlsen E, Andersson AM, Petersen JH, Skakkebaek NE. History of febrile illness and variation in semen quality. Hum Reprod. 2003; 18(10):2089-92.

21 Okanlawon A, Dym M. Effect of chloroquine on the formation of tight junctions in cultured immature rat sertoli cells. J Androl. 1996 May;17(3):249-55.

22 Boretti A. Favipiravir use for SARS CoV-2 infection. Pharmacol Rep. 2020;72(6):1542-52.

23 Ma L, Xie W, Li D, Shi L, Mao Y, Xiong Y, et al. Effect of SARS-CoV-2 infection upon male gonadal function: a single center-based study. MedRxiv. 2020.

24 Rastrelli G, Di Stasi V, Inglese F, Beccaria M, Garuti M, Di Costanzo D, et al. Low testosterone levels predict clinical adverse outcomes in SARS-CoV-2 pneumonia patients. Andrology. 2021;9(1):88-98.

25 Schroeder M, Tuku B, Jarczak D, Nierhaus A, Bai T, Jacobsen $\mathrm{H}$, et al. The majority of male patients with COVID-19 present low testosterone levels on admission to Intensive Care in Hamburg, Germany: a retrospective cohort study; 2020. 